\title{
Non-Cardiac Surgery in a Paediatric Patient with Permanent Pacemaker - Anaesthetic Implications
}

\author{
Authors \\ Dr A. Rajithasri ${ }^{1}$, Dr Krishna Chaithanya ${ }^{2^{*}}$, Dr Mushahida ${ }^{3}$ \\ ${ }^{1}$ Post Graduate, ${ }^{2}$ Professor, ${ }^{3}$ Professor \& HOD \\ Department of Anaesthesiology, Narayana Medical College, Nellore, India \\ *Corresponding Author \\ Dr K. Krishna Chaithanya
}

\begin{abstract}
Children with permanent pacemaker coming for non-cardiac surgeries is a rare presentation. Anaesthetic management should be planned preoperatively as per patient's medical status and careful monitoring should be done preoperatively. We hereby present a case of 9 year old female child with permanent cardiac pacemaker posted for Maxillary cyst Enucleation.

Keywords: Pacemaker, Paediatric patients, Anaesthetic implications, Complete heart block.
\end{abstract}

\section{Introduction}

Cardiac pacemakers have revolutionized the treatment of patients with cardiac conduction defects. First pacemaker was implanted in 1958, since then cardiac device therapy is under steady expansion due to its phenomenal progress in device technology and software sophistication. Treatment for complete congenital heart block entails lifelong dependence on an artificial pacing system. The number of patients requiring non-cardiac surgery with implanted pacemakers is on the rise, this inevitably results in more patients being encountered by anaesthesiologists ${ }^{[1]}$. Care of pacemaker during surgery as well as understanding its anaesthetic implications is crucial in the management of these patients ${ }^{[2]}$.

\section{Case Report}

A 9-year-old female child, weighing $20 \mathrm{kgs}$ was posted for Maxillary cyst enucleation under general anaesthesia. She was a full term born child by normal vaginal delivery. At the age of 4 years she had been taken to a local paediatrician for fever, where the child was referred to a higher centre in view of bradycardia for further evaluation. Following week, she was diagnosed with complete congenital heart block at a tertiary care centre in Vellore and conservative management was advised. The child had no other associated congenital anomalies or defects; no history of syncopal attacks, recurrent respiratory tract infections, febrile seizures and developmental delay. Child was immunized as per protocol. There was no history of past surgical exposure. On general physical examination there 
were no signs of pallor, cyanosis, clubbing and oedema. Pulse and blood pressure were $45 \mathrm{bpm}$ and 92/64 $\mathrm{mm}$ of $\mathrm{Hg}$ respectively. On systemic examination, heart sounds were normal and chest was clear bilaterally.

As a part of preoperative evaluation, child was referred to cardiologist. Significant Chronotropic incompetence of Sino atrial node with a resting heart rate of $35 \mathrm{bpm}$ and maximum heart rate of $86 \mathrm{bpm}$ which is not adequate for that age group was noted by cardiologist and pacemaker implantation was advised in order to cope up with the stress responses related to both surgery and anaesthesia during the perioperative period.

Permanent pacemaker implantation was done under Monitored anesthesia care, 10 days before the surgery. Pacemaker details: Manufacturer- St Jude, battery life 8yrs, pacing mode is VVI with a preset baseline heart rate of $60 \mathrm{bpm}$.

Routine blood investigations: Complete Blood Picture, blood Urea, serum Creatinine, serum Electrolytes and random blood sugars were within normal limits. Blood grouping and viral markers were done.

On Chest X-ray (figure 2), Pulse generator in left infraclavicular region and pacemaker lead in right ventricular apex were noted. On ECG, regular paced rhythm and a heart rate of $60 \mathrm{bpm}$ were noted. 2D ECHO - revealed the presence of pacemaker lead in right ventricle with remaining parameters being normal and EF- 61\%. Cardiologist advised that the patient could be taken for surgery under high risk consent with minimal use of electrocautery.

Table-1 : Generic codes of pacemakers[4]

$\begin{array}{ccc}\text { I } & \text { II } & \text { III } \\ \text { Pacing } & \text { Sensing } & \text { Response } \\ & & \\ \text { O }- \text { None } & \text { O }- \text { None } & \text { O }- \text { None } \\ \text { A }- \text { Atrium } & \text { A }- \text { Atrium } & \text { I }- \text { Inhibited } \\ \text { V }- \text { Ventricle } & \text { V }- \text { Ventricle } & \text { T }- \text { Triggered } \\ \mathrm{D}-\text { Dual }(\mathrm{A}+\mathrm{V}) & \mathrm{D}-\text { Dual }(\mathrm{A}+\mathrm{V}) & \text { Dual }-\mathrm{I}+\mathrm{T} \\ \mathrm{S}-\text { Simple }(\mathrm{A} \text { or } \mathrm{V}) & \mathrm{S}-\text { Simple }(\mathrm{A} \text { or } \mathrm{V}) & \end{array}$

After taking informed consent for general anaesthe -sia, patient was kept nil per oral 8 hours for solids and 2 hours for clear liquids. On day of surgery pacemaker was set to asynchronous mode (VVI to VOO) and baseline heart rate was reset from 60 bpm to 70bpm. ECG, Pulse oxymetry, Non-invasive blood pressure, Capnography and Temperature were monitored. After pre-oxygenation for $3 \mathrm{~min}$ with $100 \%$ oxygen, anaesthesia was induced with Inj.Midazolam $2 \mathrm{mg}$, Inj. Fentanyl $40 \mathrm{mcgs}$, graded doses of Inj. Propofol $30 \mathrm{mg}$, Inj. Atracurium $10 \mathrm{mg}$ and intermittent positive pressure ventilation was given for 3 mins. Patient was intubated with oral cuffed ETT size 5.0, fixed at $15 \mathrm{cms}$ and was mechanically ventilated.

Anaesthesia was maintained with Oxygen+ air+ Sevoflurane (1.2 MAC) and Inj.Atracurium $2 \mathrm{mg}$.

During intraoperative period, care was taken to maintain normotension, normocapnea and normal temperature. Bipolar cautery was used minimally. Infraorbital nerve block given with $0.25 \%$ Levobupivacaine $1.2 \mathrm{ml}$ for postoperative analgesia. Following completion of surgery, patient was reversed with Inj.Glycopyrrolate $0.2 \mathrm{mg}+$ inj.Neostigmine $1 \mathrm{mg}$. Patient was extubated after obtaining adequate consciousness, recovery of muscle tone and power. She was shifted to I.C.U where pacemaker was reprogrammed to VVI mode with baseline heart rate $60 \mathrm{bpm}$. Care was taken to avoid hypoxia, shivering, nausea, vomiting and adequate analgesia was provided. Perioperative period was uneventful. 
Figure 1

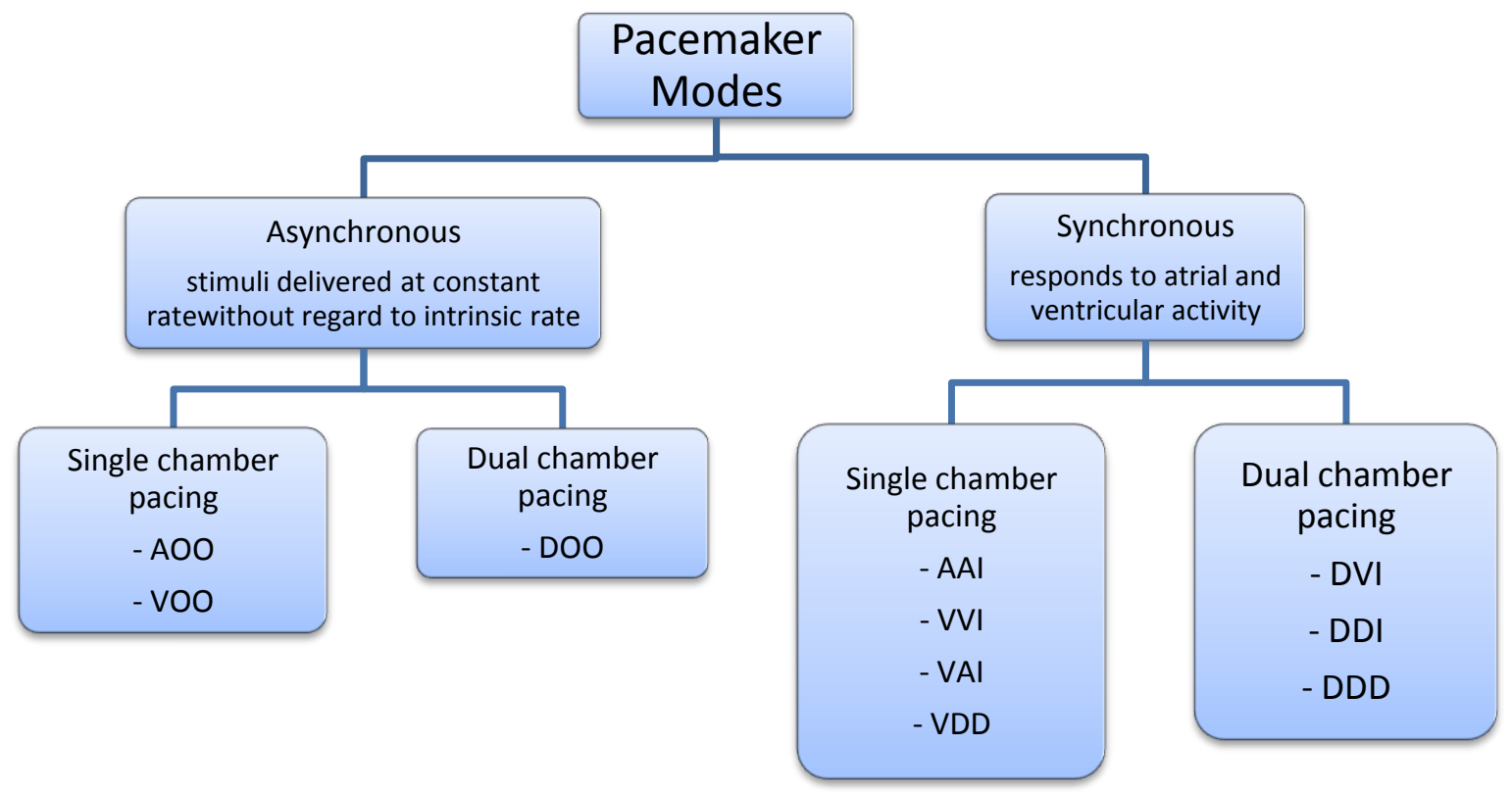

Figure 2

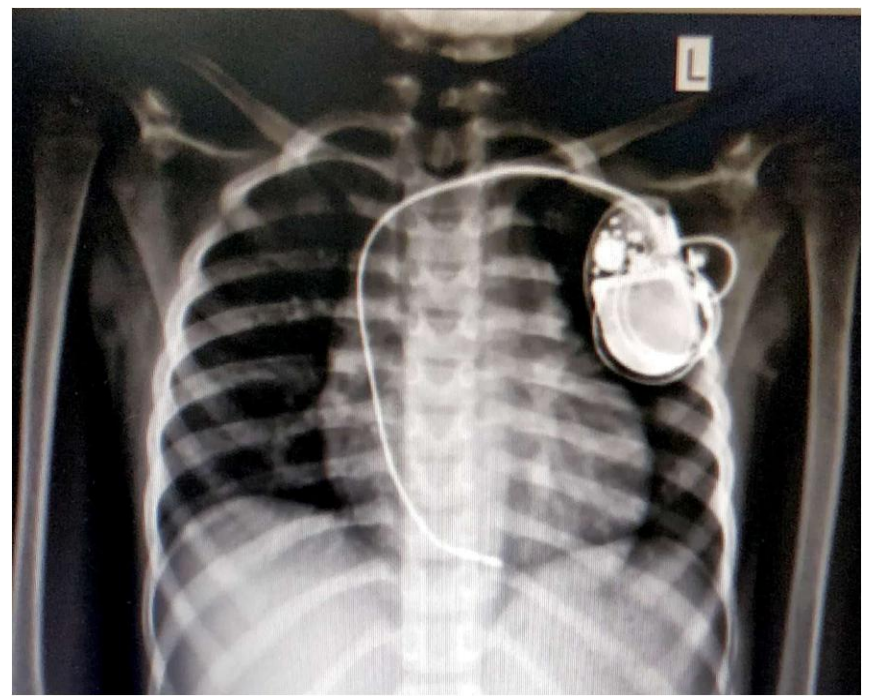

Table -2: Factors affecting pacing threshold ${ }^{[5]}$.

\begin{tabular}{|c|c|}
\hline Increase & Decrease \\
\hline 1-4 weeks after implantation & Increased catecholamines \\
Hypothermia, Hypothyroidism & Stress,Anxiety, \\
Hyperkalaemia, Acidosis/alkalosis & Sympathomimetic \\
Antiarrythmics(IA/B,2) & drugs,Anticholinergics, \\
Antiarrythmics(Ic,3) & Glucocorticoids \\
Severe hypoxia/hypoglycaemia & Hyperthyroidism \\
Inhalation-local anaesthetics & Hypermetabolic status \\
\hline possibly increase thresholds & \\
\hline
\end{tabular}

** conflicting evidence, probably dose related

\section{Discussion}

Important indications ${ }^{[3]}$ for pacemaker include symptomatic Sinus node disease, symptomatic AV nodal disease, Long QT syndrome, Hypertrophic obstructive cardiomyopathy, Dilated cardiomypathy. In this patient pacemaker was implanted for complete congenital heart block.

A Pacemaker consists of an impulse generator and a lead or leads to carry the electrical impulse to the patient's heart. Leads can be unipolar/ bipolar/ multipolar connected to the right atrial appendage for atrial pacing and right ventricular apex for ventricular pacing through vena cava or on surface of heart epicardially. Unipolar leads seem to be more sensitive to the effects of electromagnetic interference and produce larger spikes on recorded ECG. Generic codes ${ }^{[4]}$ for pacemaker are mentioned in table 1.

Pacing Threshold ${ }^{[5]}$ is the minimum amount of energy required to consistently cause depolarization and therefore contraction of the heart. It is measured in terms of both amplitude and duration for which it is applied to the myocardium. The amplitude is programmed in volts $(\mathrm{V})$ or in milliamperes in some 
devices, and the duration is measured in milliseconds. Factors affecting the myocardial pacing threshold are listed in table 2 .

In this patient pacemaker initially was pacing in VVI mode ${ }^{[2]}$ which is the most widely used form of pacing in which ventricle is sensed and paced. It senses the intrinsic $\mathrm{R}$ wave and thus inhibits the pacemaker function. This type of pacemaker is indicated in a patient with Complete heart block with chronic Atrial flutter, Atrial fibrillation and long ventricular pauses. Single chamber ventricular pacing is not recommended for patients with sinus node disease, as these patients are more likely to develop the Pacemaker syndrome.

Pacing mode was changed to VOO mode ${ }^{[2]}$ during perioperative period which is the simple form of fixed rate pacemaker which discharges at a preset rate irrespective of the inherent heart rate. However, the problems associated with asynchronous mode are that it competes with the patient's intrinsic rhythm and results in induction of tachyarrythmias. Continuous pacing wastes energy and also decreases the half-life of the battery. Other modes of pacing were shown in figure 1.

Preoperative evaluation includes evaluation of the patient and the pacemaker. Preoperative assessment should aim to identify the type of device, including details of the manufacturer and model, the programmed settings ${ }^{[2]}$. Along with routine investigations, chest x-ray and ECG, measurement of serum electrolytes (especially $\mathrm{K}+$ ) should be performed. If there is hyperkalaemia, increased current density would be required to raise the Resting membrane potential to the membrane threshold potential, making it more difficult for the pacemaker to initiate myocardial contraction.

The anaesthetic technique should be used according to the need of the patient. We used graded Propofol for induction. Etomidate and ketamine should be avoided as these cause myoclonic movements[6]. Succinylcholine should not be used as the myogenic electrical activity associated with muscle fasciculation induced by Succinylcholine may result in Electromagnetic interference ${ }^{[7]}$. We used Sevoflurane for maintenance of anaesthesia as inhalational agents do not alter current voltage thresholds of pacemaker.

Pacemaker syndrome and pacemaker failure(2) are the two major complications which may occur in these patients. Pacemaker syndrome results from a complex interaction of haemodynamic, neurohumoral and vascular changes induced by the loss of AV synchrony. Pacemaker failure may be due to generator failure, lead failure, or failure to capture

Spontaneous device failure is uncommon but there are a number of potential hazards in the medical environment, the most important of which is electromagnetic interference (EMI). The use of electrocautery during surgery is a well-recognized hazard $^{[8,9]}$. EMI may produce signals that might inhibit the pacing stimuli or trigger ventricular pacing due to atrial oversensing. Additionally, current generated by the electrocautery can cause myocardial damage due to concentration of current at the electrode-tissue interface and subsequent elevation of pacing threshold. Use of the electrocautery tip close to the Pacemaker may cause it to revert to a noise reversion fixed rate mode or inhibit it due to oversensing of signals ${ }^{[10]}$.

As this was an elective procedure, we had requested for stand by cardiologist, and pacemaker programmer (magnet) was also available throughout the perioperative period. We allowed the use of bipolar cautery as it is less hazardous than unipolar $^{[11]}$. If unipolar cautery is to be used, the grounding plate should be placed in a path as far away as possible from the site of pacemaker, so that pacemaker should not be in the same path of current. Electrocautery should not be used within 15 $\mathrm{cm}$ of pacemaker. Frequency of electrocautery should be limited to 1 second bursts in every 10 seconds to prevent repeated Asystolic periods- short bursts with long pauses of cautery are preferred. 
Continous monitoring of pulsatile perfusion (finger on the pulse) is mandatory to confirm that electrical activity is converted to mechanical systoles. In the present case, factors affecting pacing threshold were avoided and perioperative course was uneventful. Pacemaker settings are to be re-programmed back to original setting post operatively ${ }^{[12]}$.

\section{Conclusion}

Pacemaker device does require some special attention. Anticipation of behaviour, both of the patient and of the device, is the key to safe management for an uncomplicated procedure.

Anaesthetic management should be planned preoperatively as per patient's medical status.

Careful intraoperative monitoring of ECG, pulse oximetry and blood pressure should be done. Electrocautery needs to be used with precaution for minimum EMI. Provision of temporary pacing should be available in Operation Theatre to deal with emergency situation of pacemaker malfunctioning. Pacemaker should always be rechecked after the procedure.

\section{References}

1. T.V.Salukhe, D. Dob, R. SuttonBr J Anesth 2004; 93:95-104.

2. Rastogi S, Goel S, Tempe DK, Virmani S. Anaesthetic management of patients with cardiac pacemakers and defibrillators for non-cardiac surgery. Annals of cardiac anaesthesia 2005; 8: 21-32.

3. Gregoratos G, Abrams J, Epstein AE, et al. ACC/AHA/NASPE 2002. Guideline update for implantation of cardiac pacemaker and Antiarrhythmia devices- Summary article (a report of the ACC/ AHA/NASPE committee to update the 1998 pacemaker guidelines) $J$ Am Coll Cardiol2002; 40: 1703-1719.

4. Hayes DL, Zipes DP. Cardiac pacemakers and cardioverter-defibrillator. In: Braunwald
E, Heart Disease6th edition, Philadelphia, WB Saunders, 2001; 7775-814.

5. Atlee JL, Cardiac pacing and electroversion. In: Kaplan JA, ed Cardiac Anesthesia, 4th edition Philadelphia, WB Saunders, 1999; 959-89.

6. Senthuran S, Toff WD, VuylstekeA, Solesbury PM, Menon DK. Implanted cardiac pacemakers and defibrillators in anaesthetic practice. $\mathrm{Br} \mathrm{J}$ Anaesth2002; 88:627-31.

7. Finfer SR. Pacemaker failure on induction of anaesthesia.Br J Anaesth 1991; 66: 50912.

8. Irnich W. Pacemaker related patient mortality. Pace1999; 22: 1279-83.

9. Levine PA, Balady GJ, Lazar HL, Belott PH, Robert AJ. Electrocautery and pacemakers: management of the paced patient subject to electrocautery. Ann Thorac Surg 1986; 41: 313-7.

10. Okan Erdogan, Electromagnetic Interference on Pacemakers. Indian Pacing and Electrophysiology Journal (ISSN 09726292), 2(3): 74-78 (2002).

11. Ramon-Gonzalez J, Hyberger LK, Hayes DL. Is electrocautery still a clinically significant problem with contemporary technology. Pace 2001; 24: 709.

12. ASA Task Force Anaesthesiology 2005; 103:186- 98 . 